\title{
State-Oriented Productivity Analysis in One-of-a-Kind-Production
}

\author{
Florian Tietze" and Hermann Lödding \\ Hamburg University of Technology, Hamburg, Germany \\ \{florian.tietze, loedding\}@tuhh. de
}

\begin{abstract}
Traditional productivity analysis has emerged in mass production and cannot be adopted one-to-one on One-of-a-kind production (OKP). Due to the non-repetitive character of the processes in OKP, productivity improvements do not reproduce like in mass production. In addition, preparatory activities such as orientation, material handling and positioning usually consume a lot more time than the actual value-adding activities in OKP. Therefore, OKP requires analysis methods that deliver: 1) a generic working cycle to enable repetitive productivity improvements; 2) activities of personnel in production processes, which include the preparatory activities. We introduce a state-oriented approach for productivity measurement in OKP. With a case study we show how to capture, visualize and evaluate state data of an OKP.
\end{abstract}

Keywords: one-of-a-kind production, productivity analysis, lean management.

\section{Introduction}

Methods of lean production facilitate a systematic approach and have enabled large productivity gains primarily in mass production [1]. In one-of-a-kind productions, the use of many methods of lean production is hardly practicable because the processes rarely repeat [2]. The materials, sequence, location, necessary tools as well as the position of parts are changing significantly more often in an OKP. That is why the percentage of manual activities is higher. Analyzing and improving the productivity of these labor-intensive processes is a crucial task for these companies [3].

This paper presents a methodology that enables companies with OKP to analyze the influencing factors of the labor productivity with relatively low effort. The focus is on data collection. This is based on the definition of worker activities, objects and a generic working cycle and enables the aggregation of productivity data across various departments and processes. The analysis of the data allows to compare the different factors of productivity and to prioritize improvement activities. An industrial example shows that the method is suitable to analyze OKP and the influences on the labor productivity in detail. The method claims to be valid for universal OKP, although the case is taken from the maritime industry.

\footnotetext{
* Corresponding author.
} 


\section{One-of-a-Kind Production}

Production as well as the assembly can be divided according to different types. In this context, mass production and one-of-a-kind production are the two extremes [4].

Within mass production, products are highly standardized. All relevant information is available from the start of production. As a result of the many repetitions, process improvements lead to high productivity growth.

The OKP with its highly customized product needs a high flexibility of production processes and high reconfigurability of the used equipment. The following organizational principles are typically used [5]: manual production workshop, construction site principle, flexible manufacturing cells. The OKP is characterized by several features [6]: i.e. higher secondary times, long lead times, low transparency of business processes, complex production control and more.

These properties of the OKP lead to low productivity, which is reflected in a different structure of the working time. In mass production, workers spend a huge amount of time directly at the product. In OKP, workers spend an essential time for the acquisition of information and organization of work patterns.

Due to this focus, many methods of lean production cannot be applied directly. Moreover, the lack of transparency as well as the less detailed and not standardized work processes makes it more difficult to analyze the productivity. The various types of manufacturing in the OKP, the parallel processes and the complex production control complicate the prioritization and implementation of improvement activities.

The following section provides an overview of different approaches aiming at the analysis of productivity in industrial companies.

\section{Productivity Analysis}

A productivity analysis examines a production process normally at defined time intervals to detect productivity potentials [7]. Different approaches for productivity analysis exist:

- Empirical productivity analysis: Productivity indices [8], econometric models [9] and methods of linear programming [10] belong to the empirical productivity analysis. Overall, these approaches are not effective in the OKP, because they provide abstract results based on theoretical assumptions and the heterogeneous causes of lost productivity cannot be identified or associated with the losses.

- Operative methods: Methods-Time Measurement [11], SMED [12] and PrimarySecondary-Analysis [13] provide a high level of detail. However, for the OKP this productivity analyses are limited, because the operations are not sufficiently planned in detail and the processes are repeated rarely.

This overview shows the lack of productivity analysis in OKP, since most of the methods serve rather abstract level and often require high data acquisition and operational effort. Thus, productivity analysis in the OKP has to fulfill the following requirements: 
- The productivity analysis must be flexible in different types of production.

- Losses during preparation and other ancillary activities must be included.

- The methodology should enable repeatable productivity gains.

- The results of the analysis should allow prioritization of improvement actions.

\section{Concept of a State-Oriented Productivity Analysis}

Based on the requirements described for productivity analysis in OKP the procedure of a state-oriented productivity analysis will be explained. These efficiency or productivity analysis have mainly been applied to machines or interlinked manufacturing systems $[14,15]$. The worker states need to cover the whole time span of paid labor time to realize a comprehensive analysis. Value-adding tasks as well as non-valueadding tasks can equally be a source for reduced labor productivity [16]. Fig. 1 shows the methodology for the productivity management in OKP.

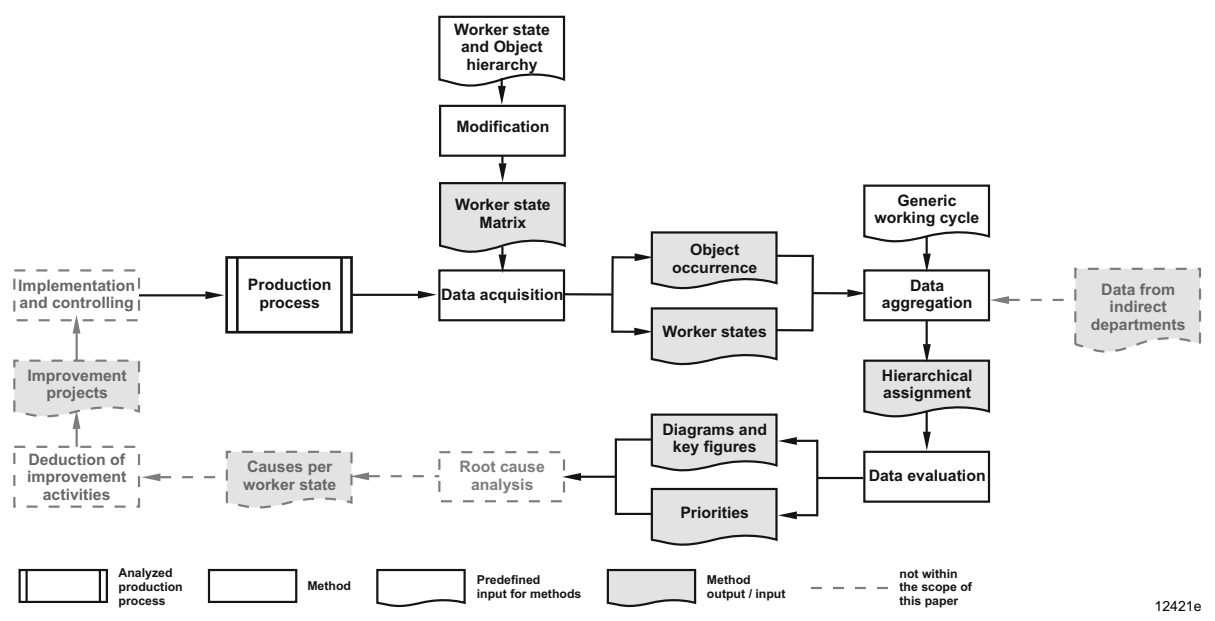

Fig. 1. Methodology for productivity management in OKP

It combines various sub-methods, which are linked with inputs and outputs. The scope of this paper is limited to the first four sub-methods: Modification, data acquisition, data aggregation and data evaluation. In order to describe the operation of the methodology, the following section describes the predefined inputs and methods.

\subsection{Worker Activity and Object Hierarchy}

Mixed production types, few standard activities and higher secondary and setup times make it difficult to describe the operational procedure in OKP in a standardized way. Fruehwald developed a method to standardize flexible set-up processes [17]. In this method, one process step is fully described by combining an object with an activity.

The result is a worker activity and object hierarchy as shown in fig. 2 . 


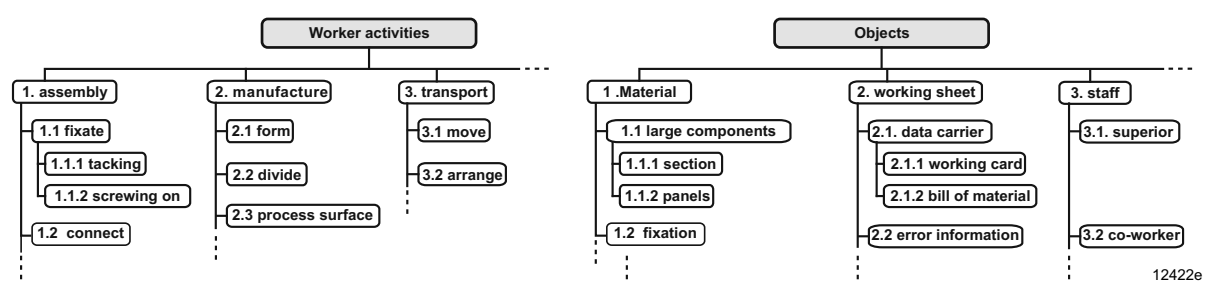

Fig. 2. Example of the worker activity and object hierarchy

The worker activities are clustered in groups and structured with a numerical logic. To identify the worker activities and to minimize the data acquisition effort, each worker activity has an individual number, which implies the main and sub group. The same logic structures the object hierarchy. As a result each unique worker state can be described with a combination of an activity and an object.

\subsection{Modification and Worker State Matrix}

The combination of the worker activity and object hierarchy is a generic worker activity and object matrix. A plausibility check helps to reduce the potential amount of combinations. There are two reasons for an invalid activity/object combination:

- Technical and logical inconsistency: E.g., it is not possible to screw a colleague.

- Definitive inconsistency: It is invalid to screw a section, because small parts like clamps are screwed on large parts.

It may be necessary to modify the matrix for the data acquisition, because the worker activities are universally valid and have to complement only in special cases. However, the objects have to be adapted to the use case, but it is possible to use generic matrices for industries. The tailored matrix is the input for the data acquisition.

\subsection{Data Acquisition}

Acquiring time data in OKP is not common, because of the long operation times and low degree of detail of the process descriptions. For the acquisition of above described state data, different approaches have been defined:

- Work sampling measures the frequency of occurrence of certain predefined events by using short-time observations at predetermined times in the investigation area [18]. The results can be aggregated and evaluated statistically.

- Time studies enable a detection of detailed work processes without interrupting the worker. In contrast to work sampling, time study measure actual times.

Prior to the data acquisition, an area has to be selected. Useful criteria are the head count or the repetition frequency of the process due to the large amount of paid working time or rather the potential by gaining repetitive productivity improvements. The observed area and processes determine the data acquisition method: Work samplings 
are used in manual working shops or productions cells with transparent overview; time studies suit the construction site principle with generic processes, which repeat often. The above described and modified matrix defines the state categories.

To gain efficient productivity improvements the result of the data acquisition has to be combined with a repetitive and generic working cycle.

\subsection{Generic Working Cycle}

The aim of the generic working cycle is to enable repeatable improvements. As in mass production, repetitions allow an efficient use of improvement activities and lean methods. From the perspective of the worker in an OKP environment the working process can be determined in five steps as shown in fig. 3:

Information $\left.\left.\sum \begin{array}{c}\text { Material } \\ \text { allocation }\end{array}\right\rangle \begin{array}{l}\text { Component } \\ \text { preparation }\end{array}\right\rangle$ Execution $\left.\sum \begin{array}{c}\text { Post } \\ \text { processing }\end{array}\right\rangle$

Fig. 3. Generic Working Cycle

At the information phase the worker clarifies the work task and allocates all required information. The next step is to gather necessary materials and tools. During the component preparation phase, the worker sets up machines and builds auxiliary equipment. When everything is prepared, the worker can execute his work. This is the only value-adding activity. At the end the worker has to clean the work area, bring tools back and document results. The single phases of the generic working cycle are defined as operational fields. These fields facilitate a goal-orientated approach to prioritize improvement activities and support the deduction of standard methods.

To gain repetitive productivity improvements, the output from the data acquisition has to be aggregated to the described generic working cycle.

\subsection{Data Aggregation}

The paid working time consist of different time portions (equation 1). The duration of worker absence consists mainly of times for vacation, training or illness. This information has to be gathered from the corporate data system. The attendance can be recorded with the defined worker state matrix. The classification inside the attendance time is carried out manually and supported by two principles:

- The predefined worker activities have clear classifications specified in the matrix. For example: reading is always in the operation field Information.

- Ambiguous worker activities can be assigned to operation fields by considering the objects. For example: the combination of the worker activity searching and the object engineering drawings is part of the operation field Information; in combination with object drill this worker state belongs to the operation field Material allocation.

If each worker state is classified by these two guidelines, the output from the data acquisition can be aggregated and assigned to the operation fields. 


$$
T_{\text {paid }}=T_{a t t}+T_{a b s}+T_{N R}
$$

$T_{\text {paid }}=T_{a t t} * \sum_{j=1}^{5} O F_{j}+T_{a b s}+T_{N R} \quad$ with $\quad O F_{j}=\frac{\sum_{i=1}^{n} s_{j}}{n} \quad s_{j} \in O F_{j}$

$\begin{array}{llll}\mathrm{T}_{\text {paid }} & \text { paid working time [hrs] } & \mathrm{T}_{\mathrm{abs}} & \text { absence time [hrs] } \\ \mathrm{T}_{\text {att }} & \text { attendance time [hrs] } & \mathrm{T}_{\mathrm{NR}} & \text { not recorded activities [hrs] } \\ \mathrm{OF}_{\mathrm{j}} & \text { Portion of all worker states of the operational field } \mathrm{j}[\%] \\ \mathrm{s}_{\mathrm{j}} & \text { Observation of worker state in the operation field } \mathrm{j} \text { during work sampling [-] } \\ \mathrm{j} & \text { Index for different operational fields [-] } \\ \mathrm{n} & \text { Number of observations during the work sampling [-] }\end{array}$

If time studies are used, the sum of the worker state durations per operation field plus a term for absence times and not recorded activities equals the paid working time. The work sampling method provides the ratio of worker states and can be converted to the paid time through multiplication with $\mathrm{T}_{\text {paid }}$ (equation 2).

The result of the data aggregation is a database of all worker states in the observed area. If required, it can be extended with additional information.

\subsection{Data Evaluation}

The state data needs to be processed and illustrated using key figures and diagrams. The evaluation allows to determine priorities and to deduct methods for operational fields. There are two ways of evaluation:

- Prioritization of the greatest operational field: The combination of worker activities with the greatest portion and most used objects facilitates the prioritization of productivity losses and references for a detailed root analysis.

- Case-based improvement: If a process is recorded with the worker activities state matrix, it is possible to use this as a basis for a standard working description using the generic working cycle.

Key figures and diagrams allow to determine priorities, to derive improvement potentials and standardize work based on worker activity and object hierarchy.

\section{Case Study}

The proposed method was applied to an OKP at a shipyard. The worker state hierarchy was tailored on the basis of working plans and interviews. The final matrix included 62 different as well as 80 different objects. A plausibility check reduced the number of combinations to round about 1100 different worker states.

Accordingly, the two data acquisition modes were assigned: Work sampling was done for the pre-outfitting and the outfitting at the berth; a time study was conducted at the prefabrication. A recording list with defined fields for activities, objects, time and notes was used and merged with a software tool. 
The data acquisition required an effort of two eight-hour days and additional eight hours for tailoring the worker activity and object hierarchy and preparation of the recording lists. The time study described a 3 hours working process. Fig. 4 shows the allocation of the operational fields for the working sample and the time study.

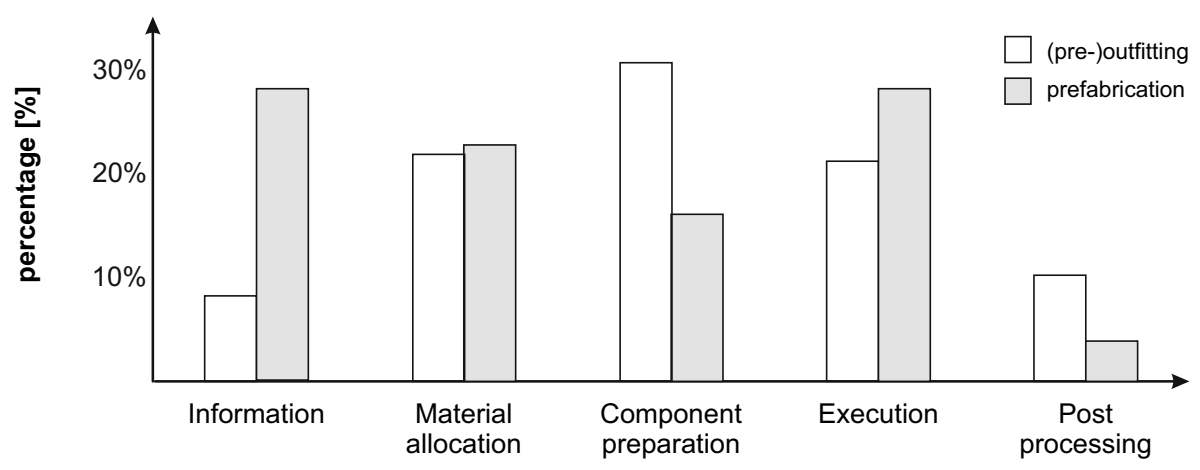

Fig. 4. Allocation to operational fields

As expected the workers spent a big amount of their working time with preparative activities. The evaluation results are not representative, due to of the small amount of samples. However, the case study proves the functionality and practicability of the developed concept especially in different types of production. The evaluation proved the applicability of the worker activity and object hierarchy: Only four worker states out of 87 samples could not be clearly identified and the observer had to ask the worker. All process steps could be described with the worker state matrix.

One challenge is to locate the worker at the construction principle, which was used at the outfitting. In $24 \%$ of the samples the worker was not detected in a time frame of one Minute. Also, the distance between the observation points is very long. This problem can be solved by using self reports or auxiliary utilities like location boards.

An evaluation with a significant amount of samples is planned to demonstrate the connection from allocation of the operational fields to productivity potential.

\section{Conclusion and Outlook}

The paper has introduced a method for a state-oriented productivity analysis in OKP. It combines worker activities and objects with operational fields of a generic working cycle to gain repetitive improvements. With the proposed method, production managers can improve transparency of productivity potentials with low effort.

The case demonstrated the enhanced possibilities of a worker state matrix based on activities and objects. This tool is suitable to describe the work process also in the different production types of OKP in a standardized way.

The ongoing studies at the IPMT include the definition of an accurate sample size for working samples. An improved software demonstrator will further reduce the effort for the data acquisition. In addition, another data acquisition method like self-reports will be adapted for this methods and evaluated. 
Furthermore, the evaluation has to be done with further industrial partners to cover different types of productions. A next research step will be to link the distribution of the operational fields with standard optimization. The case showed the high demand on adapted or new improvement methods for OKP especially in the operational fields gathering information and preparing the components and working place.

Acknowledgement. The presented work was done in cooperation with a shipyard within the research project PROSPER, funded by the German Federal Ministry of Economics and Technology (Bundesministerium für Wirtschaft und Technologie BMWi / Projektträger Jülich PTJ) due to a decision of the German Bundestag.

\section{References}

1. Roos, D., Womack, J., Jones, D.: The Machine That Changed the World: The Story of Lean Production. Harper Perennial (1991)

2. Salem, O., Solomon, J., Genaidy, A., Minkarah, I.: Lean Construction: From Theory to Implementation. J. Manage. Eng. 22(4), 168-175 (2006)

3. Ohno, T.: Toyota Production System - Beyond Large-Scale Production. Productivity Press, Cambridge (1988)

4. Gruß, R.: Schlanke Unikatfertigung - Zweistufiges Taktphasenmodell zur Steigerung der Prozesseffizienz in der Unikatfertigung. Gabler Verlag, Wiesbaden (2010)

5. Wiendahl, H.-P.: Betriebsorganisation für Ingenieure. Hanser, Munich (2008)

6. Piller, F.T.: Mass Customization. Wiesbaden (2006)

7. Nebl, T.: Produktivitätsmanagement. Hanser, Munich (2002)

8. Craig, C.E., Harris, R.C.: Total Productivity Measurement at the Firm Level. Sloan Management Review 14(3), 13-29 (1973)

9. Sudit, E.F.: Productivity measurement in industrial operations. European Journal of Operational Research 85(3), 435-453 (1995)

10. Charnes, A., Cooper, W.W., Rhodes, E.: Measuring the efficiency of decision making units. European Journal of Operational Research 2(6), 429-444 (1978)

11. Bokranz, R., Landau, K.: Produktivitätsmanagement von Arbeitssystemen. SchäfferPoeschel, Stuttgart (2006)

12. Shingo, S.: A Revolution in Manufacturing: The SMED System. Productivity Press, Cambridge (1985)

13. Lotter, B., Spath, D., Baumgartner, P.: Primär-Sekundär-Analyse: Kundennutzenmessung und Kundennutzenorientierung im Unternehmen. Expert Verlag, Renningen (2002)

14. Wiendahl, H.P., Hegenscheidt, M.: Produktivität komplexer Produktionsanlagen. Zeitschrift für Wirtschaftlichen Fabrikbetrieb 96(4), 160-163 (2001)

15. Grando, A., Turco, F.: Modelling plant capacity and productivity: conceptual framework in a single-machine case. Production Planning \& Control 16(3), 309-322 (2005)

16. Czumanski, T., Lödding, H.: Integral analysis of labor productivity. In: 45th CIRP Conference on Manufacturing Systems, Procedia CIRP, vol. 3, pp. 55-60 (2012)

17. Frühwald, C.: Analyse und Planung produktionstechnischer Rüstabläufe. VDI-Verlag, Düsseldorf (1990)

18. Simons, B.: Das Multimoment-Zeitmeßverfahren. Univ., Cologne and Dortmund (1987) 